J. Lake Sci. (湖泊科学), 2012, 24(4):615-622

http: //www.jlakes.org. E-mail: jlakes@niglas.ac.cn

(C) 2012 by Journal of Lake Sciences

\title{
贵州省麦岗水库沉积物磁性特征及其与粒度的关系”
}

\author{
李春梅, 王红亚 ${ }^{* *}$ \\ ( 北京大学城市与环境学院,北京 100871)
}

\begin{abstract}
摘 要: 对贵州省麦岗水库沉积物环境磁性特征的研究表明,亚铁磁性矿物主导了沉积物矿物磁性特征,但同时也存在 反铁磁性矿物等其他矿物, 超顺磁颗粒在沉积物中广泛存在. 在所选矿物磁性参数中, $\chi_{\mathrm{If}} \chi_{\mathrm{fd}} 、 S O F T$ 与粒度不相关; $\chi_{\mathrm{ARM}}$ 、 $S I R M 、 F_{300}$ 与粒度相关,但相关系数不高; $\chi_{\mathrm{ARM}} / \chi_{\mathrm{If}} \chi_{\mathrm{ARM}} / S I R M$ 和粒度显著相关, 可以作为粒度的代用指标. 研究结果显 示, 磁性参数确实可以作为粒度的代用指标. 但对比研究表明, 在不同沉积环境, 甚至相似沉积环境的沉积物中, 矿物磁 性参数和粒度的关系可能不同, 在特定沉积环境中, 利用磁性参数作为粒度的代用指标应该在充分研究的基础上进行, 使研究结果更为可靠.
\end{abstract}

关键词: 磁性特征;粒度;沉积物;麦岗水库

\section{Magnetic properties and their relationships with particle size of sediments from Maigang Resevoir, Guizhou Province, China}

\author{
LI Chunmei \& WANG Hongya \\ (College of Urban and Environmental Sciences, Peking University, Beijing 100871, P. R. China)
}

\begin{abstract}
Three sediment cores were extracted from Maigang Reservoir, located in the southwest of Guizhou Province, China. Ferrimagnetic minerals are the main magnetic minerals of the sediments. There are also other magnetic minerals, for instance, antiferromagnetic component. Super-paramagnetic grains were widely observed in the sediments. There is no significant relationship between mineral magnetic parameters, $\chi_{\mathrm{lf}}, \chi_{\mathrm{fd}}, S O F T$ and textural parameters. Mineral magnetic parameters, $\chi_{\mathrm{ARM}}, S I R M, F_{300}$ is correlated with textural parameters but with low correlation coefficients; Given the significant correlations between $\chi_{\mathrm{ARM}} / \chi_{\mathrm{If}}, \chi_{\mathrm{ARM}} /$ SIRM and textural parameters, $\chi_{\mathrm{ARM}} / \chi_{\mathrm{If}}$, and $\chi_{\mathrm{ARM}} / S I R M$ can be used as a proxy of particle size. Results presented here do indicate that mineral magnetic parameters can be used as a particle size proxy. These data and other studies on different environments demonstrate that the relationship between mineral magnetic parameters and textual properties can vary from different sedimentary environments even in the same overall sedimentary system, and in some circumstances, mineral magnetic parameters are not suitable to be particle size proxy. To employ magnetic characteristics as a particle size proxy, the mechanism behind the relationship between magnetic parameters and particle size should be explored fully for each sedimentary environment.
\end{abstract}

Keywords: Magnetic properties; particle size; sediment; Maigang Reservoir

环境磁学是一门介于环境科学、地学和磁学之间的边缘学科 ${ }^{[1]}$. 由于矿物磁性测量具有简便、敏感、经 济、快速、需要样品量少及无破坏等优点, $1970 \mathrm{~s}$ 以来,在地学的很多领域得到广泛应用 ${ }^{[2]}$. 相关研究主要集 中于利用岩石、土壤、沉积物等的矿物磁性特征提取环境污染、古气候及古环境演变、物源判别等信息 ${ }^{[3-9]}$. 已有研究表明,矿物磁性是研究沉积物来源、沉积物运移路径和作为地球化学、放射物质、有机质含量及粒 度代用指标的重要工具 ${ }^{[10-12]}$. 其中, 对于沉积物矿物磁性与粒度的关系及两者在环境变化研究中的应用受 到很多学者的关注 ${ }^{[13-15]}$. 通常认为, 沉积物粒度对沉积物的其他相关指标 (如沉积物中自然和人为污染物含 量、放射性物质、矿物磁性特征等)有着重要的影响 ${ }^{[16]}$. 这主要是因为沉积物组成越细,其表面积越大, 表面

* 国家重点基础研究发展计划 “973” 项目 (2003CB415201) 和科技部基础性工作专项课题项目 (2007FY140800-1) 联合 资助. 2011-05-17 收稿;2011-10-10 收修改稿. 李春梅,女, 1985 年生, 博士研究生; E-mail: lichunmei023@ 126. com.

** 通信作者;E-mail:why@ urban.pku.edu.cn. 
电荷及阳离子交换能力越强, 从而使沉积物有更强的化学吸附能力; 反之, 沉积物组成越粗, 化学吸附能力 相对越弱 ${ }^{[16]}$. 由于粒度对沉积物的矿物磁性特征具有重要影响, 结合矿物磁性测量的优点, 许多学者认为矿 物磁性特征具有作为粒度代用指标的潜力, 并对此开展了一系列研究 ${ }^{[17-19]}$. 在对海洋、湖泊、河口、河流等沉 积物的矿物磁性特征与粒度的关系进行分析的基础上,认为通常沉积物中较高的矿物磁性与细颗粒的含量 呈正相关,而与粗颗粒的含量呈负相关,但在不同区域不同研究中, 特定矿物磁性参数所代表的粒级具有一 定的差异 ${ }^{[16]}$. 因此, 为使矿物磁性参数作为粒度的代用指标更有普适性和代表性,需对不同沉积环境中的沉 积物进行充分的研究. 喀斯特地区是生态环境脆弱的特殊区域, 而目前对该区域沉积物的矿物磁性特征及 其与粒度的关系研究较少.

本文以位于我国西南喀斯特地区的贵州省麦岗水库为研究区域,在研究其沉积物矿物磁性特征的基础 上,探讨该水库沉积物特定矿物磁性参数与不同粒度组成的关系, 从而丰富对矿物磁性特征作为粒度代用 指标的研究,对增强其代表性具有一定的意义.

\section{1 研究区概况}

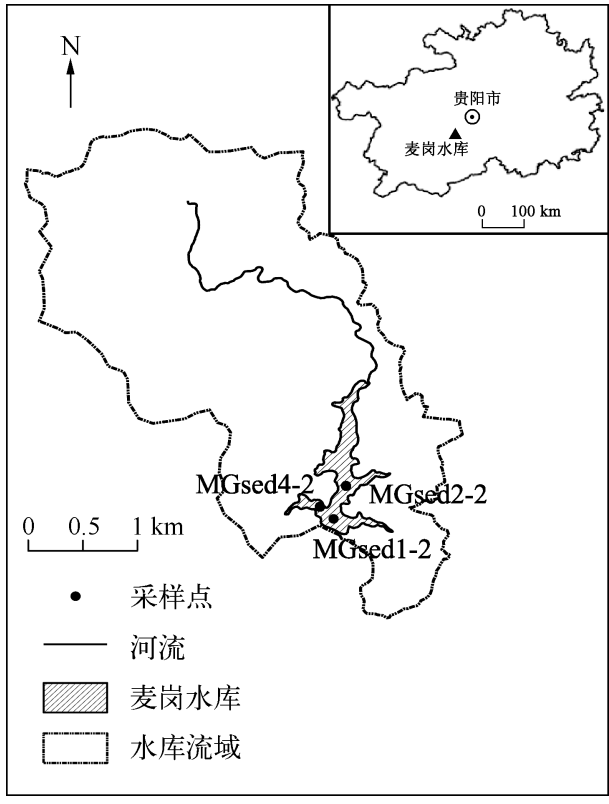

图 1 麦岗水库采样点及其汇水流域

Fig. 1 Sampling sites of Maigang Reservoir and its catchment
麦岗水库 $\left(26^{\circ} 00^{\prime} 50^{\prime \prime} \mathrm{N}, 106^{\circ} 17^{\prime} 38^{\prime \prime} \mathrm{E}\right)$ 地处贵州省西南 部紫云县东北的坝羊乡, 位于珠江流域格凸河二级支流洛 河上游 (图 1$)^{[20]}$. 水库修建于 1956 年, 以大气降水补给 为主, 多年平均年降水量为 $1337 \mathrm{~mm}$, 基本没有人库河流, 库尾有一条较小的溪流, 坝址以上积水面积约为 $7.5 \mathrm{~km}^{2}$, 多年平均径流量为 $0.17 \mathrm{~m}^{3} / \mathrm{s}$. 是一座以灌溉为主, 兼养 鱼、饮水等综合功能的蓄水水库.

水库所在位置属于亚热带季风湿润气候带, 地带性植 被为亚热带常绿阔叶林, 流域内土壤以黄壤、石灰土为主, 水稻土次之,喀斯特地貌发育,地形起伏相对较小. 流域内 人类活动以农业为主, 工业活动极少, 主要的土地利用类 型为耕地, 且多为旱地, 水田较少.

\section{2 材料和方法}

2008 年 4 月,在麦岗水库使用中国科学院贵阳地球 化学研究所研发的 SWB-1 型重力采样器取得 3 个沉积物 柱芯, 分别是长度为 $15 \mathrm{~cm}$ 的 MGsed1-2(26 $06^{\circ} 31^{\prime \prime} \mathrm{N}, 106^{\circ}$ $\left.17^{\prime} 35^{\prime \prime} \mathrm{E}\right) 、$ 长度为 $13 \mathrm{~cm}$ 的 MGsed2-2(26 $00^{\circ} 35^{\prime \prime} \mathrm{N}, 106^{\circ} 17^{\prime}$ $\left.36^{\prime \prime} \mathrm{E}\right)$ 和长度为 $7 \mathrm{~cm}$ 的 MGsed4-2(26 $\left.20^{\prime} 35^{\prime \prime} \mathrm{N}, 106^{\circ} 17^{\prime} 31^{\prime \prime} \mathrm{E}\right)$. 沉积物柱芯取出水面时未受扰动, 水一泥界面清晰, 样品 保存完好. 柱芯采出后, 现场用分样器以 $1 \mathrm{~cm}$ 为一个样品

厚度自上而下进行取样, 将所得样品分别装人干净的 $50 \mathrm{ml}$ 离心管中密封保存, 共计 35 个样品.

样品的矿物磁性参数测量在北京大学地表过程分析与模拟教育部重点实验室进行. 样品经离心、冷冻 干燥后, 取出适量用玛瑙研钵轻磨成粉状, 装人 $2 \mathrm{~cm} \times 2 \mathrm{~cm} \times 2 \mathrm{~cm}$ 塑料盒中并压实密封, 使用英国 Bartington MS2 磁化率仪测试低频磁化率 $\left(\chi_{\mathrm{If}}, 0.47 \mathrm{kHz}\right)$ 和高频磁化率 $\left(\chi_{\mathrm{hf}}, 4.7 \mathrm{kHz}\right)$, 计算得到频率磁化率 $\left(\chi_{\mathrm{fd}} \%\right.$, $\left.\chi_{\mathrm{fd}} \%=\left(\chi_{\mathrm{lf}}-\chi_{\mathrm{hf}}\right) / \chi_{\mathrm{lf}} \times 100\right)$; 将样品置于交变磁场峰值为 $90 \mathrm{mT}$, 直流场为 $0.04 \mathrm{mT}$ 下的 Molspin 交变退磁仪 中, 样品获得非滞后剩磁 $(A R M)$, 利用旋转磁力仪测得样品的 $A R M$, 计算获得非磁滞剩磁磁化率 ( $\chi_{\mathrm{ARM}}, \chi_{\mathrm{ARM}}$ $=A R M / 0.3814)$; 将样品依次置于 $20 、 300$ 和 $1000 \mathrm{mT}$ 磁场强度下的 Molspin 脉冲磁化仪中, 样品获得相应的 等温剩磁, 然后, 使用 Molspin 旋转磁力仪测定样品的 $I R M_{20 \mathrm{mT}} 、 I R M_{300 \mathrm{mT}}$ 和 $I R M_{1000 \mathrm{mT}}$, 其中, $I R M_{20 \mathrm{mT}}$ 为 “软” 剩磁 $(S O F T), I R M_{1000 \mathrm{mT}}$ 为饱和等温剩磁 $(S I R M)$. 同时, 计算 $\chi_{\mathrm{ARM}} / \chi_{\mathrm{lf}} \backslash \chi_{\mathrm{ARM}} / S I R M$ 和 $F_{300}\left(F_{300}=I R M_{300 \mathrm{mT}} / S I R M \times\right.$ $100)$ 比值. 以上磁性参数中, $\chi_{\mathrm{If}} \chi_{\mathrm{ARM}} 、 S O F T$ 和 $S I R M$ 主要与磁性矿物含量有关; $\chi_{\mathrm{fd}} \chi_{\mathrm{ARM}} / \chi_{\mathrm{If}} \chi_{\mathrm{ARM}} / S I R M$ 和 $F_{300}$ 主要反映磁性矿物的颗粒大小和类型 ${ }^{[10]}$. 各参数及其指示意义见表 1 . 
表 1 主要磁性参数及指示意义 ${ }^{[21-23]}$

Tab. 1 Magnetic parameters used in the study and their basic interpretation

\begin{tabular}{|c|c|}
\hline 参数 & 指示意义 \\
\hline 低频磁化率 $\chi_{\mathrm{II}} /\left(\times 10^{-8} \mathrm{~m}^{3} / \mathrm{kg}\right)$ & 通常用作亚铁磁性矿物含量的粗略度量, 突出代表 SP 和 MD 颗粒含量 \\
\hline 频率磁化率 $\chi_{\mathrm{fd}} / \%$ & 可以反映样品中超顺磁 (SP) 颗粒的存在和相对含量 \\
\hline 非磁滞剩磁磁化率 $\chi_{\mathrm{ARM}} /\left(\times 10^{-8} \mathrm{~m}^{3} / \mathrm{kg}\right)$ & 一般指示单畴 $(\mathrm{SD})$ 的亚铁磁性矿物含量 \\
\hline 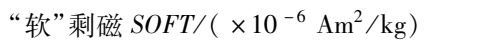 & 指示亚铁磁性矿物, 特别是多畴 (MD) 和假单畴 (PSD) 亚铁磁性颗粒含量 \\
\hline 饱和等温剩磁 $S I R M /\left(\times 10^{-6} \mathrm{Am}^{2} / \mathrm{kg}\right)$ & $\begin{array}{l}\text { 样品获得的最大剩磁, 反映等温剩磁主要贡献者 (亚铁磁性矿物和反铁磁 } \\
\text { 性矿物) 的含量, 常用来表示样品中磁性矿物的总量 }\end{array}$ \\
\hline$\chi_{\mathrm{ARM}} / \chi_{\mathrm{If}}$ & $\begin{array}{l}\text { 指示亚铁磁性矿物颗粒的大小, 较高值反映稳定单畴 (SSD) 颗粒, 而较低 } \\
\text { 值则显示了较多的多畴或超顺 (SP) 磁颗粒 }\end{array}$ \\
\hline$\chi_{\mathrm{ARM}} / \operatorname{SIRM} /\left(\times 10^{-2} \mathrm{~m} / \mathrm{A}\right)$ & $\begin{array}{l}\text { 反映磁性颗粒的大小, 较高的比值指示较细的磁性颗粒, 在稳定单畴颗粒 } \\
\text { 的比值最高, 随颗粒变粗迅速降低 }\end{array}$ \\
\hline$F_{300} / \%$ & $\begin{array}{l}\text { 指示样品中亚铁磁性矿物 (如磁铁矿) 与不完整反铁磁性矿物 (如针铁 } \\
\text { 矿、赤铁矿) 的相对比例, 随着不完整反铁磁性矿物贡献的增加而降低 }\end{array}$ \\
\hline
\end{tabular}

粒度测定在中国科学院地理科学与资源研究所完成, 实验仪器为英国 Malvern Instruments 公司生产的 Mastersizer 2000 激光粒度仪. 实验步骤为: 将干燥后的样品进行充分混合, 去除植物根系, 取适量样品放人烧 杯中; 加人 $30 \%$ 的 $\mathrm{H}_{2} \mathrm{O}_{2}$ 至过量以充分去除样品中的有机质; 溶液加热至沸腾后静置冷却; 向溶液加人 $10 \%$ 的 $\mathrm{HCl}$ 去除 $\mathrm{CaCO}_{3}$; 用 $5 \%$ 的六偏磷酸钠溶液进行分散, 加热至沸腾; 将烧杯注满水, 静置 $1 \mathrm{~d}$ 后, 抽去上清液 以洗去 $\mathrm{HCl}$, 反复此步骤, 直至溶液变为中性; 用激光粒度仪测试样品粒度.

\section{3 结果与讨论}

\section{1 沉积物矿物磁性特征}

各矿物磁性参数在不同沉积物柱芯之间, 以及同一柱芯的不同样品之间存在差异 (表 2 , 图 2). 如 SOFT 变化于 $229.1 \times 10^{-6} \sim 1572.3 \times 10^{-6} \mathrm{Am}^{2} / \mathrm{kg}$ 之间, SIRM 变化于 $984.1 \times 10^{-6} \sim 3694.8 \times 10^{-6} \mathrm{Am}^{2} / \mathrm{kg}$ 之间, $\chi_{\mathrm{ARM}} / S I R M$ 的极小值和极大值分别为 $0.05 \times 10^{-2} 、 0.15 \times 10^{-2} \mathrm{~m} / \mathrm{A}$, 相差约 $3 \sim 7$ 倍. 就平均值来看, 各柱芯 之间相差不大, 如 $\chi_{\mathrm{If}} \chi_{\mathrm{fd}} \chi_{\mathrm{ARM}} / \chi_{\mathrm{If}}$ 及 $F_{300}$ 等参数的平均值相差很小, 说明麦岗水库沉积物矿物磁性特征的差 异性和共性. 对于三个沉积物柱芯的磁性特征存在差异的原因,有待进一步研究.

表 2 麦岗水库不同沉积物柱芯矿物磁性参数

Tab. 2 Mineral magnetic properties for different sediment cores of Maigang Reservoir

\begin{tabular}{|c|c|c|c|c|c|c|c|c|c|}
\hline \multirow{2}{*}{ 磁性参数 } & \multicolumn{3}{|c|}{ MGsed1 -2 } & \multicolumn{3}{|c|}{ MGsed2-2 } & \multicolumn{3}{|c|}{ MGsed4-2 } \\
\hline & 极小值 & 极大值 & 均值 & 极小值 & 极大值 & 均值 & 极小值 & 极大值 & 均值 \\
\hline$\chi_{\mathrm{lf}} /\left(\times 10^{-8} \mathrm{~m}^{3} / \mathrm{kg}\right)$ & 26.9 & 35.2 & 30.3 & 16.1 & 31.1 & 30.3 & 23.3 & 47.8 & 31.6 \\
\hline$\chi_{\mathrm{fd}} / \%$ & 7.2 & 11.4 & 9.2 & 4.3 & 9.6 & 7.7 & 5.5 & 12.0 & 8.9 \\
\hline$\chi_{\mathrm{ARM}} /\left(\times 10^{-8} \mathrm{~m}^{3} / \mathrm{kg}\right)$ & 165.7 & 257.8 & 186.3 & 99.6 & 263.9 & 147.9 & 149.7 & 218.8 & 178.6 \\
\hline $\mathrm{SOFT} /\left(\times 10^{-6} \mathrm{Am}^{2} / \mathrm{kg}\right)$ & 359.5 & 453.0 & 414.8 & 229.1 & 438.2 & 316.9 & 357.8 & 1572.3 & 602.8 \\
\hline $\operatorname{SIRM} /\left(\times 10^{-6} \mathrm{Am}^{2} / \mathrm{kg}\right)$ & 1206.6 & 1604.1 & 1352.3 & 1361.9 & 1873.3 & 984.1 & 1148.0 & 3694.8 & 1925.4 \\
\hline$\chi_{\mathrm{ARM}} / \chi_{\mathrm{If}}$ & 5.3 & 8.1 & 6.2 & 5.0 & 9.1 & 6.8 & 3.9 & 6.8 & 5.7 \\
\hline$\chi_{\mathrm{ARM}} / \operatorname{SIRM} /\left(\times 10^{-2} \mathrm{~m} / \mathrm{A}\right)$ & 0.12 & 0.16 & 0.14 & 0.08 & 0.16 & 0.11 & 0.05 & 0.15 & 0.09 \\
\hline$F_{300} / \%$ & 92.0 & 95.8 & 93.6 & 90.2 & 96.1 & 93.3 & 91.8 & 100.0 & 95.2 \\
\hline
\end{tabular}

$F_{300}$ 变化于 $90.2 \% \sim 100.0 \%$ 之间, 三个沉积物柱芯的平均值均在 $93 \%$ 以上, 即经过 $300 \mathrm{mT}$ 磁场磁化 后, 沉积物样品的剩磁已接近饱和, 表明亚铁磁性矿物主导了样品的矿物磁性特征, 但同时不完整的反铁磁 性矿物对磁性特征也有贡献. 三个沉积物柱芯之间的 $F_{300}$ 均值较为相近, 说明沉积物柱芯间存在相对一致的 磁性矿物组成. $\chi_{\mathrm{If}}$ SSOFT 与 SIRM 的关系表明 (已剔除 SIRM 的两个极端值), $\chi_{\mathrm{If}}$ 和 SOFT 均与 SIRM 呈正相 


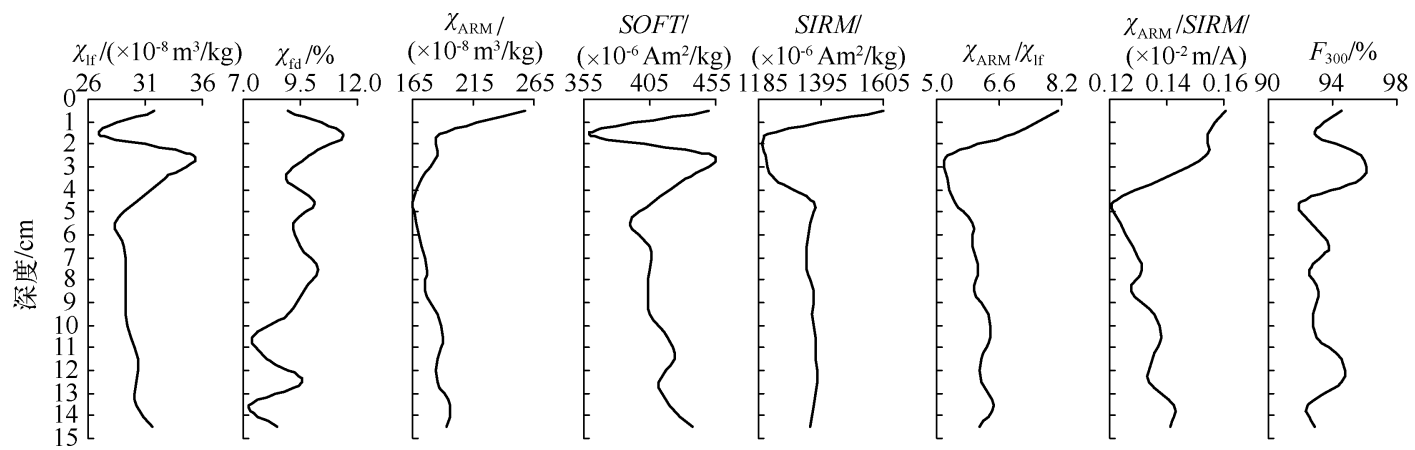

MGsed1-2
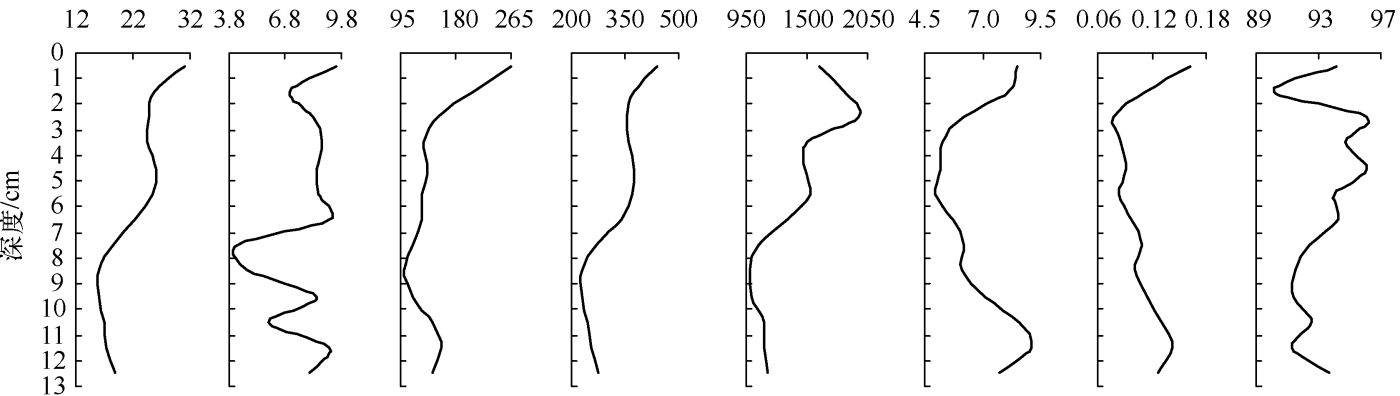

MGsed2-2
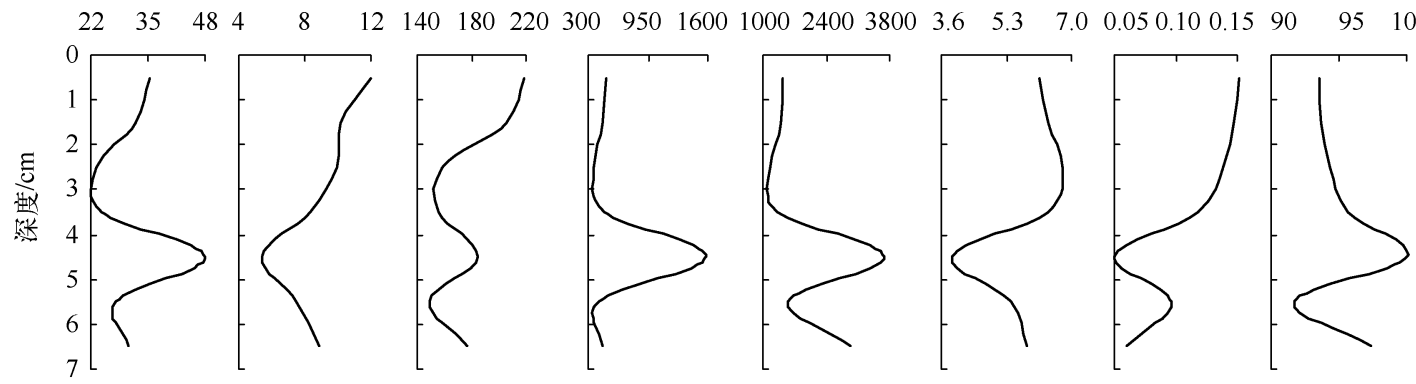

图 2 麦岗水库沉积物柱芯矿物磁性的垂向变化

Fig. 2 Down core variations of mineral magnetic properties for sediment cores

关, 相关系数分别为 0.56 和 0.63 (图 3), 说明亚铁磁性矿物对矿物磁性特征有很大贡献, 但不完整反铁磁 性矿物对磁性矿物特征的贡献也不容忽视.
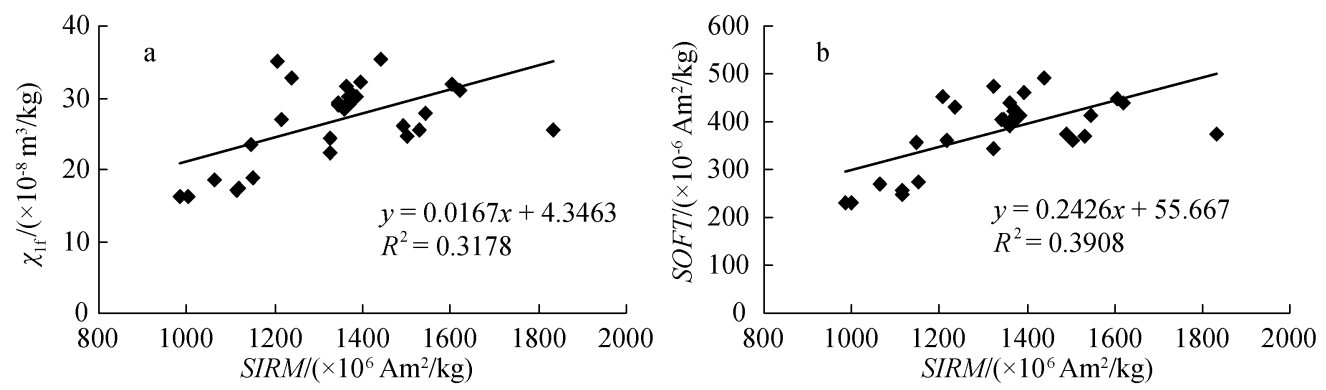

图 $3 S I R M$ 与 $\chi_{\mathrm{If}}(\mathrm{a})$ 及 $\operatorname{SOFT}(\mathrm{b})$ 的相关关系

Fig. 3 Correlation relationships between SIRM and $\chi_{\mathrm{If}}(\mathrm{a}), \operatorname{SIRM}$ and SOFT (b) 
样品的 $\chi_{\mathrm{fd}}$ 均大于 $3 \%$,三个沉积物柱芯样品的均值分别为 $9.2 \% 、 7.7 \%$ 和 $8.9 \%$, 显示了 SP 颗粒的存 在. MGsed1-2 和 MGsed4-2 柱芯的 $\chi_{\mathrm{fd}}$ 分别在 7.2\% 11.4\%、5.5\% 12\% 之间, 表明样品 SP 颗粒含量相对较 高. $\chi_{\mathrm{ARM}} / S I R M$ 相对较高, 大于 $5 \times 10^{-4} \mathrm{~m} / \mathrm{A}$, MGsed1-2 和 MGsed2-2 样品的值大于 $12 \times 10^{-4} \mathrm{~m} / \mathrm{A}$, 表明沉积 物中存在丰富的细颗粒亚铁磁性物质. $\chi_{\mathrm{ARM}} / \chi_{\mathrm{If}}$ 值较低, 均小于 10 , 可能是 SP 颗粒所致.

\section{2 沉积物粒度组成与矿物磁性特征的关系}

沉积物的粒度测试结果表明, 三个沉积物柱芯平均粒径均显示沉积物粒度由细变粗再变细的垂向变化 (图 4). 通过测试及计算可知, 所测沉积物样品的粘土 $(<4 \mu \mathrm{m})$ 含量的变化范围为 $30.8 \% \sim 80.0 \%$, 平均含 量为 $51.0 \%$. 粉砂 $(4 \sim 63 \mu \mathrm{m})$ 含量的变化范围为 $19.1 \% \sim 68.9 \%$, 平均含量为 $48.2 \%$. 其中, 极细粉砂 $(4 \sim$ $8 \mu \mathrm{m}$ ) 含量在 $15.1 \% \sim 27.1 \%$ 之间, 平均含量为 $22.8 \%$; 细粉砂 $(8 \sim 16 \mu \mathrm{m})$ 含量在 $4.0 \% \sim 25.5 \%$ 之间, 平 均含量为 $16.3 \%$; 中粉砂 $(16 \sim 32 \mu \mathrm{m})$ 含量在 $0 \sim 17.2 \%$ 之间, 平均含量为 $7.3 \%$; 粗粉砂 $(32 \sim 63 \mu \mathrm{m})$ 含量 在 $0 \% \sim 8.4 \%$ 之间, 平均含量为 $1.8 \%$. 砂 $(>63 \mu \mathrm{m}$ ) 含量的变化范围为 $0.3 \% \sim 5.0 \%$, 平均含量为 $0.8 \%$. 可 见,麦岗水库沉积物样品的粘土含量相对偏多, 砂含量相对偏少.

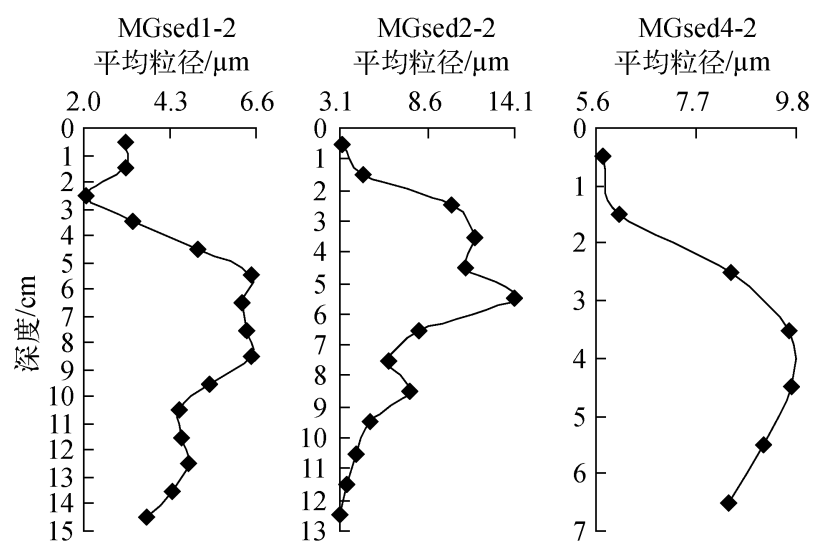

图 4 麦岗水库沉积物柱芯平均粒径

Fig. 4 Mean grain-size for sediment cores of Maigang Reservoir

对沉积物样品的矿物磁性参数和粒度特征进行相关分析, 结果表明 $\chi_{\mathrm{lf}}$ 与粒度的关系较为复杂, 除了与 $<2 \mu \mathrm{m}$ 粒级的相关系数为 0.47 , 而且该相关系数的显著性水平不到 $95 \%$, 与其他粒级均无显著相关性(表 3 ). 因此在此研究区域, 用 $\chi_{\mathrm{If}}$ 作为粒度的代用指标不合适. $\chi_{\mathrm{fd}}$ 与 $<2 、<4 、<8 、<16$ 和 $<32 \mu \mathrm{m}$ 粒级呈正相 关, 与其他粒级均呈负相关, 但各粒级的相关性均不高. 可见, 此处 $\chi_{\mathrm{fd}}$ 不能作为粒度的代用指标. $\chi_{\mathrm{ARM}}$ 与平均 粒径、 $16 \sim 32 、 32 \sim 63 、 4 \sim 63 \mu \mathrm{m}$ 的粒度组成呈负相关; 与 $<2 、<4 、<8 、<16 、<32 \mu \mathrm{m}$ 的粒度组成呈正相 关, 正负相关系数的绝对值在 $0.4 \sim 0.5$ 之间. 从粒度的三个等级看, $\chi_{\mathrm{ARM}}$ 与粉砂 $(4 \sim 63 \mu \mathrm{m})$ 、砂 $(>63 \mu \mathrm{m})$ 含量呈负相关, 与粘土 $(<4 \mu \mathrm{m})$ 含量呈正相关. 虽然相关系数不高, 但总体而言, $\chi_{\mathrm{ARM}}$ 与较粗的颗粒呈负相 关, 与较细的颗粒呈正相关. SOFT 与各粒级的关系较为复杂, 且相关系数均不高, 因此, 此处 $S O F T$ 不能用作 粒度的代用指标. $S I R M$ 与 $<2 、 2 \sim 4 、<4 、<8 、<16 \mu \mathrm{m}$ 粒级呈负相关, 与平均粒径、 $8 \sim 16 、 16 \sim 32 、 32 \sim 63$ 、 $4 \sim 63 \mu \mathrm{m}$ 粒度组成呈正相关, 但相关系数均不大于 0.5 . 可以看出, SIRM 与粘土含量呈负相关, 与粉砂含量 呈正相关, 与砂含量相关性不高. 总体上看, 研究区域沉积物 SIRM 与较细颗粒含量呈负相关, 与较粗颗粒呈 正相关, 但与 $>63 \mu \mathrm{m}$ 的粗颗粒含量相关性不显著. $F_{300}$ 与各粒级的关系与 SIRM 极为相似, 仅相关系数略有 不同. $\chi_{\mathrm{ARM}} / \chi_{\mathrm{lf}}$ 与粒度组成的相关性相对上述其他参数较为明显, 与 $<2 、 2 \sim 4 、<4 、<、<16 、<32 \mu \mathrm{m}$ 较细粒 度组成呈正相关, 与平均粒径、 $8 \sim 16 、 16 \sim 32 、 32 \sim 63 、 4 \sim 63 、>63 \mu \mathrm{m}$ 较粗粒度组成呈负相关. 从粒度划分 的三个等级看, $\chi_{\mathrm{ARM}} / \chi_{\mathrm{If}}$ 与粘土含量呈正相关, 相关系数为 0.52 , 与粉砂含量呈负相关, 相关系数为 -0.5 , 与 砂含量呈负相关, 但相关系数不高, 仅为 $-0.22 \cdot \chi_{\mathrm{ARM}} / S I R M$ 与各粒级的相关性是所选参数中最高. $\chi_{\mathrm{ARM}} /$ $S I R M$ 与 $<32 \mu \mathrm{m}$ 的各个粒级呈正相关, 与平均粒径、 $4 \sim 63 \mu \mathrm{m}$ 及 $>8 \mu \mathrm{m}$ 的各个粒级呈负相关. 可见, $\chi_{\mathrm{ARM}}$ ' 
SIRM 与粘土含量呈正相关, 与粉砂含量和砂含量呈负相关, 但与砂含量的相关系数相对较低. 因此, $\chi_{\mathrm{ARM}} /$ $S I R M$ 与较细颗粒含量呈正相关, 与较粗颗粒含量呈负相关. 可以看出, $\chi_{\mathrm{ARM}} / \chi_{\mathrm{If}}$ 和 $\chi_{\mathrm{ARM}} / S I R M$ 与各粒级的关 系相似,但前者比后者的相关系数低.

表 3 沉积物样品矿物磁性参数与粒度的相关系数

Tab. 3 The correlation coefficients between mineral magnetic parameters and particle size of sediment samples

\begin{tabular}{ccccccccc}
\hline 粒度 & \multicolumn{1}{c}{$\chi_{\mathrm{If}}$} & \multicolumn{1}{c}{$\chi_{\mathrm{fd}}$} & $\chi_{\mathrm{ARM}}$ & SOFT & SIRM & $\chi_{\mathrm{ARM}} / \chi_{\mathrm{If}}$ & $\chi_{\mathrm{ARM}} / S I R M$ & $F_{300}$ \\
\hline 平均粒径 & -0.05 & -0.17 & $-0.50^{* *}$ & 0.19 & $0.35^{*}$ & $-0.55^{* *}$ & $-0.78^{* *}$ & $0.35^{*}$ \\
$<2 \mu \mathrm{m}$ & 0.47 & 0.22 & $0.48^{* *}$ & -0.23 & -0.32 & $0.49^{* * *}$ & $0.70^{* *}$ & -0.26 \\
$2 \sim 4 \mu \mathrm{m}$ & -0.18 & -0.20 & 0.13 & -0.31 & $-0.44^{* *}$ & $0.44^{* *}$ & $0.57^{* *}$ & -0.33 \\
$4 \sim 8 \mu \mathrm{m}$ & -0.13 & -0.16 & -0.13 & -0.05 & -0.11 & -0.06 & 0.03 & -0.22 \\
$8 \sim 16 \mu \mathrm{m}$ & 0.10 & -0.11 & -0.28 & 0.32 & $0.38^{*}$ & $-0.44^{* *}$ & $-0.57^{* *}$ & 0.24 \\
$16 \sim 32 \mu \mathrm{m}$ & 0.05 & -0.14 & $-0.40^{*}$ & 0.30 & $0.42^{*}$ & $-0.54^{* *}$ & $-0.73^{* *}$ & $0.37^{*}$ \\
$32 \sim 63 \mu \mathrm{m}$ & -0.12 & -0.20 & $-0.53^{* *}$ & 0.10 & 0.30 & $-0.49^{* *}$ & $-0.77^{* *}$ & $0.39^{*}$ \\
$4 \sim 63 \mu \mathrm{m}$ & 0.01 & -0.18 & $-0.43^{*}$ & 0.27 & $0.38^{*}$ & $-0.50^{* *}$ & $-0.71^{* *}$ & 0.29 \\
$>63 \mu \mathrm{m}$ & -0.12 & -0.01 & -0.26 & -0.13 & -0.03 & -0.22 & -0.28 & -0.06 \\
$<4 \mu \mathrm{m}$ & 0.002 & 0.18 & $0.44^{* *}$ & -0.26 & $-0.37^{*}$ & $0.52^{* *}$ & $0.72^{* *}$ & -0.29 \\
$<8 \mu \mathrm{m}$ & -0.03 & 0.15 & $0.43^{*}$ & -0.28 & $-0.41^{*}$ & $0.55^{* *}$ & $0.75^{* *}$ & $-0.35^{*}$ \\
$<16 \mu \mathrm{m}$ & 0.02 & 0.16 & $0.47^{* *}$ & -0.23 & $-0.38^{*}$ & $0.56^{* *}$ & $0.78^{* *}$ & $-0.39^{*}$ \\
$<32 \mu \mathrm{m}$ & 0.13 & 0.16 & $0.50^{* *}$ & -0.05 & -0.24 & $0.46^{* *}$ & $0.70^{* *}$ & $-0.33^{*}$ \\
\hline
\end{tabular}

* 代表显著相关, $P<0.05 ; * *$ 代表极显著相关, $P<0.01$.

综上所述,对所选麦岗水库沉积物样品矿物磁性特征与粒度的关系分析可以看出, $\chi_{\mathrm{lf}} \chi_{\mathrm{fd}}$ 和 $S O F T$ 由于 与各粒级的相关性均不显著, 所以不能用作粒度的代用指标. $\chi_{\mathrm{ARM}}$ 与较细颗粒含量呈正相关, 与较粗颗粒含 量呈负相关, 但相关系数不是很高, 因此, 在用 $\chi_{\mathrm{ARM}}$ 作为粒度代用指标时需慎重. SIRM 和 $F_{300}$ 与粒度的关系 较为相似, 都与较粗颗粒含量呈正相关, 与较细颗粒含量呈负相关, 但相关系数也偏低, 因此用作粒度代用 指标时仍需注意. $\chi_{\mathrm{ARM}} / \chi_{\mathrm{If}}$ 和 $\chi_{\mathrm{ARM}} / S I R M$ 与粒度的关系相似, 与较细颗粒含量呈正相关, 与较粗颗粒含量呈负 相关,且相关系数较高, 可以用作粒度的代用指标.

\section{3 与其他研究结果的比较}

通过与其他区域不同沉积环境的相关研究比较,发现此区域的研究结论与其他区域具有共同点,但也 存在不同之处. Bonnett 等 ${ }^{[24]}$ 对 Wirral 和 Landcashire 海岸和河口沉积物进行矿物磁性参数和粒度关系的研 究, 以及 Clifton 等 ${ }^{[25]}$ 对 Irish 海东的盐沼和海滨泥地沉积物进行的研究,均表明 $\chi_{\text {If }} \chi_{\text {ARM }}$ SIRM 与粒度显著相 关. 砂含量与 $\chi_{\mathrm{If}}($ 相关系数 $r=-0.94) \chi_{\mathrm{ARM}}(r=-0.81)$ 呈负相关,但与 SIRM 的相关性不显著; 粉砂含量与 $\chi_{\mathrm{If}}(r=0.96) \chi_{\mathrm{ARM}}(r=0.93) 、 S I R M(r=0.96)$ 呈显著正相关; 粘土含量与 $\chi_{\mathrm{If}}(r=0.77) \chi_{\mathrm{ARM}}(r=0.92) 、 S I R M$ $(r=0.81)$ 也呈正相关. Liu 等 ${ }^{[26]}$ 根据沉积物的特点, 对所取长江口沉积物分成 4 组进行相关分析, 结果表明 某些组的样品 $\chi_{\mathrm{ARM}} \chi_{\mathrm{fd}}$ 与粘土及 $<16 \mu \mathrm{m}$ 粒级呈显著正相关; SIRM 在某些组与 $<16 、 8 \sim 16 、 2 \sim 4 \mu \mathrm{m}$ 粒级呈 显著正相关, 在某些组则与 $63 \sim 125 、 250 \sim 500 \mu \mathrm{m}$ 相关. Oldfield 等 $^{[27]}$ 对 Irish 海沉积物的研究表明, $\chi_{\mathrm{ARM}}$ 与 $<30 \mu \mathrm{m}$ 的较细粒级显著相关. 本文的研究结果与此有较大不同, 砂的含量与 $\chi_{\mathrm{If}} \chi_{\mathrm{ARM}}$ 、SIRM 均无显著相关; 粉砂含量与 $\chi_{\mathrm{If}}$ 均不相关, 与 $\chi_{\mathrm{ARM}}$ 呈负相关, 与 SIRM 呈正相关,粘土含量与 $\chi_{\mathrm{ARM}}$ 呈正相关, 与 SIRM 呈负相关 而且相关系数均不高. $\chi_{\mathrm{ARM}}$ 与 $<32 \mu \mathrm{m}$ 粒级呈正相关, 但相关系数不高. $\chi_{\mathrm{fd}}$ 与各粒级均无相关性.

Zhang 等 ${ }^{[28]}$ 对长江河口沉积物进行相关分析, 结果显示 $\chi_{\mathrm{ARM}}$ 和 $\chi_{\mathrm{fd}}$ 与 $<2 、 2 \sim 4 、 4 \sim 8 、 8 \sim 16 、<4 、<8$ 、 $<16 \mu \mathrm{m}$ 呈显著正相关, SIRM 与 $>32 \mu \mathrm{m}$ 粒级呈显著负相关, 本研究与此存在较大不同. 但该研究也显示, $\chi_{\mathrm{ARM}} / \chi_{\mathrm{If}}$ 和 $\chi_{\mathrm{ARM}} / S I R M$ 与粒度的关系和 $\chi_{\mathrm{ARM}}$ 相似, $\chi_{\mathrm{If}}$ 与 $<16 \mu \mathrm{m}$ 粒级不相关, 本文的研究结果显示, 除了 $\chi_{\mathrm{ARM}} /$ $\chi_{\mathrm{If}}$ 和 $\chi_{\mathrm{ARM}} / S I R M$ 与 $4 \sim 8 \mu \mathrm{m}$ 粒级不相关, 与 $8 \sim 16 \mu \mathrm{m}$ 粒级呈负相关外, 这 3 个参数与上述其他粒级的关系 与该研究结果较为相似,表明 $\chi_{\mathrm{ARM}} / \chi_{\mathrm{If}}$ 和 $\chi_{\mathrm{ARM}} / S I R M$ 可以作为粒度的代用指标.

Booth 等 ${ }^{[16]}$ 对 Carmarthen 海湾、Gwendraeth 河口、Gwendraeth Fach 和 Gwendraeth Fawr 河流等不同沉积 
环境的沉积物进行矿物磁性和粒度关系的分析, 结果表明在不同沉积环境, 矿物磁性与粒度的关系有所不 同. Carmarthen 海湾和 Gwendraeth 河口的沉积物, $\chi_{\mathrm{If}} \chi_{\mathrm{ARM}} 、 S I R M$ 与砂含量呈显著负相关, 与粉砂及粘土含量 呈正相关; Gwendraeth Fawr 河流沉积物的 $\chi_{\mathrm{If}} 、 S I R M$ 与砂、粉砂和粘土含量的关系与 Carmarthen 海湾和 Gwendraeth 河口沉积物的情况相反; Gwendraeth Fach 河流沉积物的 3 个磁性参数与各粒级均不相关.

以上对比结果表明,本研究与其它研究既存在共同点, 但也有不同之处, 原因可能是:一方面沉积物的 环境不同, 本研究的对象是水库沉积物, 而其它研究对象属于海洋、河口和河流沉积物, 沉积动力、沉积后次 生作用和细菌作用等都可能对结果产生影响 ${ }^{[29-33]}$, 具体原因有待进一步研究; 另一方面与样品数量相对较 少有关. 所有研究结果从不同角度说明矿物磁性参数可以作为粒度的代用指标. 但对不同沉积环境, 甚至相 似沉积环境的沉积物, 矿物磁性参数和粒度的关系可能不同, 因此在某些环境中, 矿物磁性可能不能作为粒 度的代用指标, 这在 Booth ${ }^{[32]}$ 等的研究中也得到类似的结论,运用时应该慎重.

\section{4 结论}

1) 麦岗水库沉积物中亚铁磁性矿物主导了其磁性特征, 同时也存在反铁磁性矿物等其他矿物. 超顺磁 颗粒在沉积物中广泛存在. 各沉积物柱芯矿物磁性特征存在差异的原因有待进一步研究.

2) 研究结果表明, 矿物磁性参数确实可以作为粒度的代用指标. 如不受沉积后次生作用、细菌作用等的 影响, 在本研究区域 $\chi_{\mathrm{ARM}} / \chi_{\mathrm{If}}$ 和 $\chi_{\mathrm{ARM}} / S I R M$ 可以作为粒度的代用指标.

3 ) 对比研究结果表明, 在不同的沉积环境甚至相似的沉积环境中, 沉积物的矿物磁性参数与粒度的关 系可能不同, 一些在某一沉积环境中可作为粒度代用指标的矿物磁性参数, 在其它沉积环境的沉积物中则 可能不能作为粒度代用指标.

4) 矿物磁性测量具有简便、快捷、经济、无破坏等优点, 被认为是粒度的一种理想代用指标. 但对某一特 定沉积环境, 用磁性参数作为粒度代用指标前, 应该对沉积物的矿物磁性参数与粒度的关系进行充分的研 究,使得研究更为可靠和准确.

致谢: 中国科学院贵阳地球化学研究所刘学炎、孟博博士, 北京大学孙旭波, 麦岗水库管理所所长于以立先 生在野外采样中, 中国科学院地理科学与资源研究所杨京蓉女士在粒度分析中给予了帮助, 在此谨致谢忱.

\section{5 参考文献}

[ 1 ] 张卫国, 俞立忠, 许 羽. 环境磁学研究的简介. 地球物理学进展, 1995,10(3):95-105.

[ 2 ] Thompson R, Oldfield F. Environmental magnetism. London: Allen and Unwin, 1986: 1-227.

[ 3 ] Horng CS, Huh CA, Chen KH et al. Air pollution history elucidated from anthropogenic spherules and their magnetic signatures in marine sediments offshore of Sourthwestern Taiwan. Journal of Marine Systems, 2009, 76(4) : 468-478.

[ 4 ] Wang H, Huo Y, Zeng L et al. A 42-yr soil erosion record inferred from mineral magnetism of reservoir sediments in a small carbonate-rock catchment, Guizhou Plateau, southwest China. Journal of Paleolimnology, 2008, 40 (3) : 897-921.

[ 5 ] 韩家桮, Hus JJ, 刘东生等. 马兰黄土和离石黄土的磁学性质. 第四纪研究, 1991, 11(4):310-325.

[6] Geiss CE, Zanner CW. Sediment magnetic signature of climate in modern loessic soils from the Great Plains. Quaternary International, 2007,162/163: 97-110.

[ 7 ] Hounslow MW, Morton AC. Evaluation of sediment provenance using magnetic mineral inclusions in clastic silicates: comparison with heavy mineral analysis. Sedimentary Geology, 2004, 171 : 13-36.

[ 8 ] Wang Y, Dong H, Li G et al. Magnetic properties of muddy sediments on the northestern continental shelves of China: Implication for provenance and transportation. Marine Geology, 2010, 274 : 107-119.

[ 9 ] Cioppa MT, Porter NJ, Trenhaile AS et al. Beach sediment magnetism and sources: Lake Erie, Ontario, Canada. Journal of Great Lakes Research, 2010, 36(4) : 674-685.

[10] Oldfield F, Yu L. The influence of particle size variations on the magnetic properties of sediments from the north-easternIrish Sea. Sedimentology, 1994,41: 1093-1108.

[11] Xie S, Dearing JA, Bloemandal I. The organic matter content of street dust in Liverpool, UK and its association with dust magnetic properties. Atmospheric Environment, 2000, 34(2) : 269-275.

[12] Zhang W, Yu L, Hutchinson SM. Diagenesis of magnetic minerals in the intertidal sediments of the Yangtze Estuary Chi- 
na, and its environmental significance. Science of the Total Environment, 2001, 266(1/2/3) : 169-175.

[13] Liu Q, Torrent J, Maher BA et al. Quantifying grain size distribution of pedogenic magnetic particles in Chinese loess and its significance for pedogenesis. Journal of Geophysical Research, 2005,110 : 1-7.

[14] 史 凯, 戴雪荣, 师育新等. 苏州澄湖 SC7 孔沉积物粒度特征及其古环境意义. 湖泊科学, 2009,21(5):741-748.

[15] 韩华玲,陈 静, 孙千里等. 埃及 Fraiyum 盆地沉积物中粒度和磁化率对风沙活动的指示意义. 湖泊科学, 2011,23 (2) : $303-310$.

[16] Booth CA, Walden J, Neal A et al. Use of mineral magnetic concentration data as a particle size proxy: A case study using marine, estuarine and fluvial sediments in the Carmarthen Bay area, South Wales, U. K. . Science of the Total Environmental, 2005, 347: 241-253.

［17］韩家森,姜文英,褚 骏. 黄土和古土壤中磁性矿物的粒度分布. 第四纪研究,1997,(3):281-287.

[18] Wang Y, Yu Z, Li G et al. Discrimination in magnetic properties of different-sized sediments from the Changjiang and Huanghe Estuaries of China and its implication for provenance of sediment on the shelf. Marine Geology, 2009,260 : 121 129.

[19] Zhang WG, Yu LZ. Magnetic properties of tidal flat sediments of the Yangtze estuary and its relationship with particle size. Science in China: Series D, 2003, 46(9): 954-966.

[20] 李春梅, 汪美华, 王红亚. 贵州麦岗水库沉积物的矿物磁性特征及其土壤侵蚀意义. 地理研究, 2010,29(11): 1971-1980.

[21] Banerjee SK, King J, Marvin JA. A rapid method for magnetic granulometry with applications to environmental studies. Geophysical Research Letters, 1981, 8(4) : 333-336.

[22] King JW, Banerjee SK, Marvin JA et al. A comparison of different magnetic methods for determining the relative grain size of magnetite in natural materials: some results from lake sediments. Earth and Planetary Science Letters, 1982, 59(2): 404-419.

[23] Maher BA. Magnetic properties of some synthetic sub-micron magnetites. Geophysical Journal Royal Astronomical Society, 1988, $94: 83-96$.

[24] Bonnett PJP, Appleby PG, Oldfield F. Radionuclides in coastal and estuarine sediments from Wirral and Lancashire. Science of the Total Environment, 1988, 70 : 215-236.

[25] Clifton J, McDonald P, Plater A et al. Derivation of a grain-size proxy to aid the modeling and prediction of radionuclide activity in salt marshes and mud flats of the eastern Irish sea. Estuarine, Coastal and Shelf Science, 1999, 48: 511-518.

[26] Liu S, Zhang W, He Q et al. Magnetic properties of East China Sea shelf sediments off the Yangtze Estuary: Influence of provenance and particle size. Geomorphology, 2010, 119: 212-220.

[27] Oldfield F, Richardson N, Appleby PG et al. ${ }^{241}$ Am and ${ }^{137}$ Cs activity in fine grained salt marsh sediments from parts of the N. E. Irish Sea shoreline. Journal of Environmental Radioactivity, 1993, 19: 1-24.

[28] Zhang WG, Yu LZ, Lu M et al. Magnetic approach to normalizing heavy metal concentrations for particle size effects in intertidal sediments in the Yangtze Estuary, China. Environmental Pollution, 2007, 147: 238-244.

[29] Oldfield F. Environmental magnetism: the range of applications. In: Walden J, Smith JP, Oldfield F eds. Environmental magnetism, a practical guide, Quaternary research association. Technical Guide, 1999, 6: 212-222.

[30] Oldfield F. The rock magnetic identification of magnetic mineral and grain size assemblages. In: Walden J, Smith JP, Oldfield F eds. Environmental magnetism, a practical guide, Quaternary research association. Technical Guide, 1999, 6: 98-112.

[31] Hesse P, Stolz JF. Bacterial magnetite and the Quaternary climate record. In: Maher BA, Thompson R eds. Quaternary climates, environments and magnetism. Cambridge: Cambridge University Press, 1999: 163-198.

[32] Booth CA. Sediment-Source-Linkages in the Gwendraeth Estuary, South Wales, based on mineral magnetic analyses [ PhD thesis ]. Wolver: University of Wolverhampton, 2002.

[33 ] Petermann H, Bleil U. Detection of live magnetotactic bacteria in South Atlantic deep-sea sediments. Earth and Planetary Science Letters, $1993,117(1 / 2)$ : 223-228. 